\title{
A Drosophila model to study the functions of TWIST orthologs in apoptosis and proliferation
}

\author{
M Gullaud ${ }^{1}$, R Delanoue ${ }^{1}$ and J Silber ${ }^{*, 1}$ \\ 1 Institut Jacques Monod, Tour43, 2, Place Jussieu, 75005 Paris, France \\ * Corresponding author: J Silber, Institut Jacques Monod, Tour43, 2, Place \\ Jussieu, 75005 Paris, France. Tel: +33-144-275720; Fax: +33-144-273660; \\ E-mail: silber@ccr.jussieu.fr
}

Received 6.8.02; revised 17.1.03; accepted 24.1.03 Edited by JA Cidlowsky

\begin{abstract}
The twist gene has been characterized for its role in myogenesis in several species. In addition, in mammalian cultured cells, it has been shown that twist is a potential oncogene antagonizing p53-dependent apoptosis. To study, in vivo, the role of twist in apoptosis and proliferation, we constructed transgenic Drosophila lines allowing ectopic expression of different twist orthologs. We report that: (i) Drosophila twist induces apoptosis and activates the reaper promoter, (ii) nematode twist induces arrest of proliferation without apoptosis, and (iii) human twist retains its potentialities observed in mammalian cultured cells and antagonizes Drosophila p53-dependent apoptosis. In addition, we show that human twist is able to induce cell proliferation in Drosophila. Data suggest that the pathway by which human twist antagonizes Drosophila p53 could be conserved. These transgenic lines thus constitute a powerful tool to identify targets and modifiers of human twist.

Cell Death and Differentiation (2003) 10, 641-651. doi:10.1038/ sj.cdd. 4401222
\end{abstract}

Keywords: drosophila; apoptosis; proliferation; twist, reaper, P53

Abbreviations: bHLH, basic helix-loop-helix; MTWIST, mouse TWIST; IGF-1, insulin-like growth factor 1 .

\section{Introduction}

The formation of a pluricellular organism needs the collaboration between two cellular processes: cell proliferation and cell differentiation. The basic helix-loop-helix (bHLH) proteins are transcription factors acting in various differentiation processes, either as positive or negative regulators, and play key roles in different developmental events like neurogenesis and myogenesis (for a review see Yun and Wold ${ }^{1}$ ). In general, they have been well conserved during evolution.

The twist gene encodes a bHLH factor that regulates distinct cell fate decisions in mesodermic tissues, especially during myogenesis, in species as distinct as Drosophila, nematode and human. The TWIST protein is conserved between Drosophila, Caenorhabditis elegans (C. elegans), and human, mainly at the level of the bHLH motif that contains the DNA-binding and protein-protein interaction domains. ${ }^{2,3}$ A WR motif can also be identified in human TWIST, but is less conserved in flies, and not recognizable in nematodes. ${ }^{4}$ The other domains are significantly divergent, which could explain the divergence observed in TWIST function in these different species (see Figure 1 and Castanon and Baylies ${ }^{4}$ ).

In Drosophila, the role of twist has been extensively studied. ${ }^{3,5,6}$ It was first identified based on its function during gastrulation. Later, it plays a key role in executing subdivision of the mesoderm, and regulates determination of the somatic muscle lineage. ${ }^{7}$ In late embryonic development, twist expression declines persisting only in myoblasts that will form adult muscles in later stages of development. ${ }^{8}$ These cells migrate on imaginal discs and proliferate. In pupae, twistexpressing myoblasts are involved in adult muscle formation. ${ }^{9}$ Interestingly, twist expression then fades suggesting the possibility that Drosophila TWIST is required to maintain the myoblasts in a proliferative state.

In C. elegans only one twist homolog, $h / h 8$, which encodes the CeTWIST protein, has been identified and it is expressed in mesodermal cells forming the embryonic-born enteric muscles and the postembryonic $\mathrm{M}$ mesoblast lineage..$^{10}$ The characterization of a null mutant for h/h8 allowed the identification of a requirement for $h / h 8$ in the cells where it is expressed, and, in addition, a later role in formation of nonstriated sex muscles. ${ }^{11}$ Unlike Drosophila twist null mutants, null hlh8 mutants are viable. Data indicate that in contrast to Drosophila TWIST, CeTWIST is not required for retaining myoblasts in a proliferative state. ${ }^{11}$

In Vertebrates, twist is known for its capacity to interfere with other bHLH proteins that control skeletal muscle development: MyoD, Myf5, myogenin, and MRF4. It has been shown that mouse twist (Mtwist) is able to inhibit skeletal myogenesis by blocking the activity of the myogenic bHLH and MEF2 family members. ${ }^{12,13}$ This and other results indicate that, in Vertebrates, twist is clearly an inhibitor of myogenesis. Null Mtwist mice die at about 11 days showing cranial neural tube defects, disorganization of the somites, and a high degree of apoptosis. ${ }^{14}$ In human, a role for twist in osteogenesis has been implicated as different mutations in the bHLH domain of twist are associated with the SaethreChotzen syndrome. ${ }^{15-18}$

Thus, the role of twist in myogenesis in different species seems to be well established but clearly with differences: activator role in Drosophila and on the contrary inhibitor role in Vertebrates. Nevertheless, a common feature between Drosophila and humans seems to be the expression of twist gene in proliferating tissues.

Recently, a new role for twist has been reported. Maestro et al. ${ }^{19}$ showed that twist could be considered as a potential oncogene that inhibits apoptosis induced by c-myc or activated p53. In rat cells, Myc-induced apoptosis is reduced by $50 \%$ by twist and twist can prevent cell death through a 


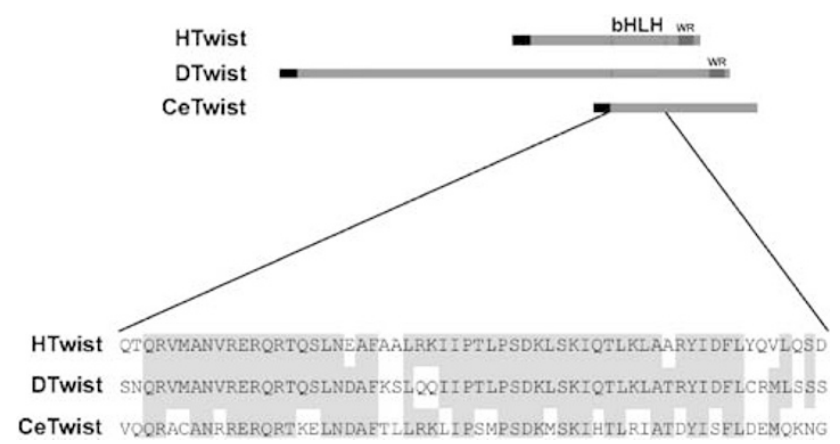

Figure 1 The different TWIST proteins. The human, nematode, and drosophila proteins differ in length, and in sequence except for the level of the bHLH domain. The NH2-terminal part of the protein is also conserved (in black), and Drosophila and human share a WR motif at the $\mathrm{COOH}$ terminus

variety of mechanisms. ${ }^{19}$ The authors suggest that the antiapoptotic properties of TWIST may result from antagonism of the p53 pathway. Indeed, Vertebrate TWIST can counteract the transcriptional activation of p53 targets like p21, MDM2, and Bax. This effect is mediated by a significant downregulation of expression of the ARF tumor suppressor gene that antagonizes the MDM2 oncogene which, in turn, is a negative regulator of $\mathrm{p} 53 .^{19}$

Furthermore, it has been shown that the insulin-like growth factor I (IGF-1) induces twist expression in NIH-3T3 cells, and this is involved in the antiapoptotic effects of the IGF-1 receptor, suggesting a possible link between the IGF-1 and p53 pathways through twist. ${ }^{20}$

These data indicate that Vertebrate TWIST is involved in processes like proliferation and p53-dependent apoptosis. This raises several questions: (i) Are these potentialities conserved by the TWIST protein of other species? (ii) Do these functions of Vertebrate TWIST exist in vivo or are they only linked to the experimental procedure used (cultured cell lines)? (iii) Is the antiapoptotic function of Vertebrate TWIST restricted to a specific cell type or is it an intrinsic capacity of the vertebrate TWIST product?

In order to address these questions, we evaluated the effects of three different TWIST proteins (drosophila, nematode, and human) in induction of apoptosis and cell proliferation. We decided to test the role of the different twist genes in the same organism, Drosophila melanogaster, inducing expression of the three transgenes ectopically by the UAS/ GAL4 system. This method has provided a powerful genetic system to elucidate cellular pathways. More particularly, it has been successfully applied to study human degenerative diseases (for review see Chan and Bonini ${ }^{21}$ ). Thus, we expressed ectopically the different TWIST proteins in a highly proliferative tissue, the wing imaginal disc, where twist is not normally expressed. This allowed us to avoid the possibility of the ectopic TWIST acting as a dominant negative with respect to the endogenous TWIST protein.

Here, we report that the ectopic expression of the three different twist genes using transgenic Drosophila lines induces a variety of responses that are very different for the three TWIST orthologs tested. This validates the use of transgenic animals expressing heterologous products to study gene function. The Drosophila twist induces apoptosis. The nematode twist induces a decrease in proliferation without apoptosis. The human twist induces both inhibition of p53-dependent apoptosis and cell proliferation. The results indicate conservation of the function of human TWIST when expressed in Drosophila and suggest possible new roles for the twist products of the other species. Moreover, they show that the p53 pathway in Drosophila responds to human twist, even if the other components of the pathway in mammals, like MDM2 and ARF, are either absent or not yet identified in Drosophila.

\section{Results}

\section{Phenotypes induced by ectopic expression of twist}

To test a possible role for twist from different species in apoptosis and proliferation, we used the UAS/GAL4 system of Drosophila. C. elegans and human transgenic lines were obtained, and will be called UAS-twistCE and UAS-twistH, respectively (see Materials and Methods). We chose to work with transgenic lines that are homozygous viable like the drosophila transgene, UAS-twistD (a gift of M Baylies).

We first tested by RT-PCR whether these transgenes are transcribed in wing imaginal discs when expressed under the control of the MS1096-GAL4 driver (see Materials and Methods and Figure 2a). These tests proved positive for all three transgenes (data not shown). For the UAS-twistH transgene, using human TWIST antibody, we determined that human TWIST presents a nuclear localization (data not shown).

We next overexpressed the different transgenes using two strong drivers, daugtherless-GAL4 (da-GAL4) and patchedGAL4 (ptc-GAL4), to evaluate their strength. UAS-twistD and UAS-twistCE gave $100 \%$ mortality with both drivers, while the UAS-twistH increased by $25 \%$ the mortality of the control (cross by Canton strain).

Finally, we ectopically expressed the different transgenes in eye and wing imaginal discs, where endogenous twist is not expressed, using eyeless-GAL4 (eye-GAL4) and GMR-GAL4 drivers for the eye and MS1096-GAL4 and vestigial-GAL4 (vg-GAL4) for the wing (Figure 1a and see Materials and Methods for the description of the drivers).

Similar strong wing phenotypes were observed for the Drosophila and $C$. elegans transgenes characterized by small size and poor differentiation (Figure $2 b-d$ ), while no effect was observed with the human transgene. This indicates that twistCE and twistD transgenes perturb normal proliferation and/or differentiation in the wing imaginal disc. Ectopic expression of either the Drosophila (Figure $2 \mathrm{e}, \mathrm{f}$ ) or nematode (Figure 2e,g) transgenes using the eye-GAL4 driver caused an almost complete disappearance of the eye structure. Again no effect was observed with the human transgene.

The effects of twist $D$ and twistCE on proliferating cells of the discs could be because of either: (i) an effect of ectopic TWIST on its own, or (ii) the formation of a heterodimer with another bHLH protein that could be necessary for cell proliferation of the wing disc, and consequent titration of this protein (a dominant negative effect). However, the high level of 
a
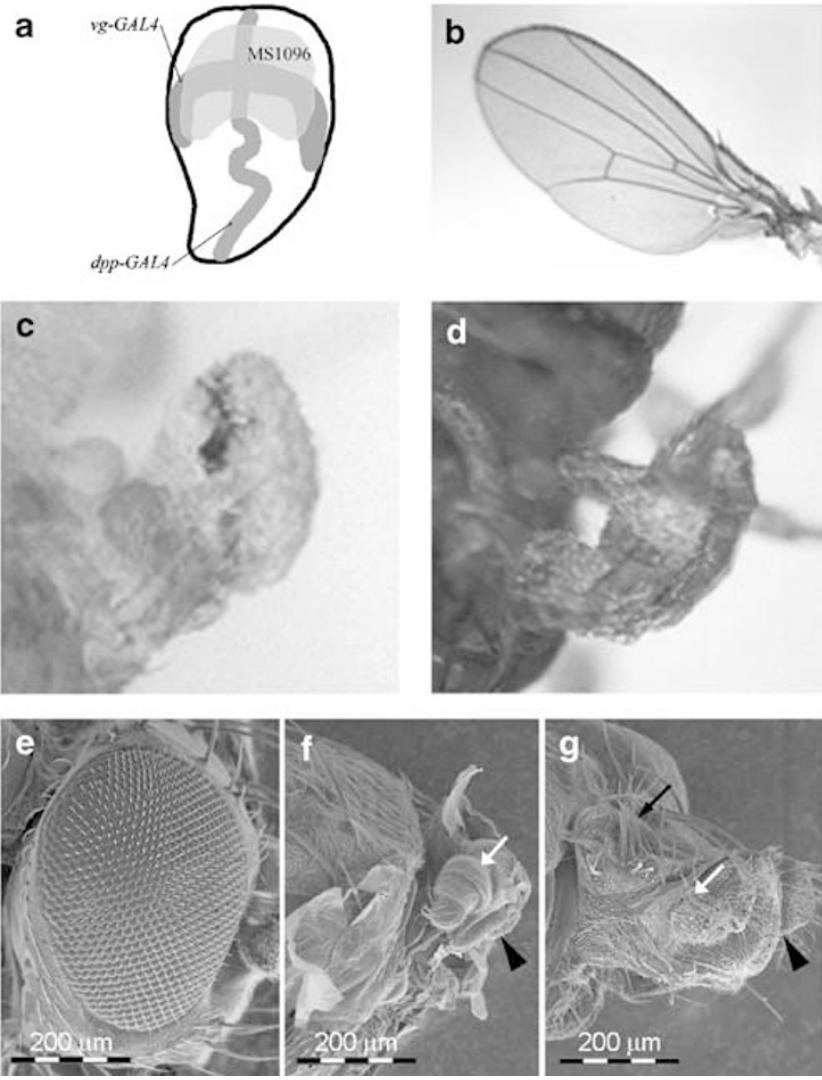

Figure 2 (a) Schematic representation of the different GAL4 drivers used to study twist ectopic expression in the wing disc. The MS1096-GAL4 driver is expressed throughout the wing pouch. It corresponds to an insertion in the Beadex locus. The $v g$-GAL4 driver allows expression according to the $v g$ wing boundary enhancer, with a high level in the part of the disc that will give the future wing margin. The dpp-GAL4 driver allows expression in the anterior compartment of the wing disc at the level of the boundary between the anteroposterior compartments. (b) Wing of an adult wild-type strain. (c) Wing of a F1 from the cross between UAS-twistD and MS1096-GAL4 flies. A strong reduction in wing size is observed. However, the wing margin is still present. (d) Wing of a F1 from the cross between UAS-twistCE and MS1096-GAL4 flies. A similar phenotype to that in $\mathrm{C}$ is observed. (e-g) Electronic microscopy of an eye and the head of adult flies. Anterior is at the right side, and dorsal face at the top. Same scale was used for each genotype: (e) Eye of wild-type strain. (f) Head of a F1 from the cross between UAS-twistD and eye-GAL4 flies. Complete disappearance of the eye is observed. Antenna (white arrow) and trunk (black arrow head) can be identified, but other head structures are absent. (g) Eye of a F1 from the cross between UAS-twistCE and eye-GAL4 flies. Antenna (white arrow) and trunk (black arrow head) can be identified. A strong reduction of the eye, identified by the specific bristles, is observed (black arrow)

expression of the transgenes and the specificity of the dimerization between the bHLH proteins argue in favor of the former hypothesis. ${ }^{22}$

\section{Ectopic expression of the Drosophila transgene induces apoptosis}

In order to understand the wing phenotype observed upon ectopic expression of the Drosophila twist transgene, we examined apoptosis in wing imaginal discs by staining with acridine orange. Using the MS1096-GAL4 driver, we ob- served a significant amount of apoptosis in the wing pouch where this driver is most strongly expressed, compared to the control (Figure $3 a, b)$. This result was confirmed using the $v g$ GAL4 driver (not shown).

To understand how the twist $D$ transgene induces apoptosis, we tested for possible induction of the reaper cell death gene. We ectopically expressed the Drosophila twist transgene using a decapentaplegic-GAL4 (dpp-GAL4), reaper$L a c Z$ strain, where $L a c Z$ is under the control of the reaper promoter. Wing discs were stained with both anti- $\beta$-galactosidase antibody and Drosophila TWIST antibody. Strong induction of reaper was observed along the length of band of the driver expression, confirming that the transgene induces apoptosis by activating reaper (Figure 3c-f). However, induction of reaper by Drosophila twist extends beyond the domain of expression of $d p p$, showing that the effect on reaper is noncell autonomous, and suggesting that reaper induction is not a direct effect of twist on the reaperpromoter. The effect of ectopic expression of twist seems to be restricted to the tissue where twist is not normally expressed. Indeed, no induction of apoptosis was observed at third larval instar when the expression of twist $D$ is directed to the myoblasts located on wing imaginal discs using the 1151-GAL4 driver (not shown). However, defects in adult thoracic muscles have been reported to occur when Drosophila twist is expressed in the wing disc myoblasts, even if the cause of these defects has not been identified yet. ${ }^{23}$

The same experiments were performed with the UAStwistCE transgene, but no apoptosis was observed when the transgene was ectopically expressed in wing imaginal discs using either the MS1096-GAL4 or the vg-GAL4 driver (data not shown). No activation of the reaper promoter was observed either (data not shown). These results exclude the possibility that the abnormal wing phenotype observed in this case was because of apoptosis. No apoptosis was observed using the UAS-twistH transgene either (data not shown).

\section{Ectopic expression of the nematode transgene induces arrest of proliferation}

To explain the wing phenotype because of the ectopic expression of the $C$. elegans twist transgene using the MS1096-GAL4 driver, we hypothesized that the phenotype could be caused by a decrease in the proliferation rate.

To test this possibility, we compared the effect of overexpression of dacapo with that of overexpression of both dacapo and twistCE on wing development. dacapo is the homolog of human p21, which inhibits cyclin-cdk complexes and thus proliferation, yet does not induce apoptosis. ${ }^{24}$ We used the vg-GAL4 driver, which allows expression at the level of the wing margin. Overexpression of dacapo using this driver causes nicks at wing tips because of absence of proliferation. ${ }^{25}$ Ectopic expression of UAS-twistCE, UASdacapo gives a stronger mutant phenotype than the ectopic expression of UAS-dacapo, UAS-LacZ(Figure 4a) suggesting that, like the dacapo gene, the ectopic expression of twistCE can affect proliferation. The UAS-dacapo, UAS-LacZ strain was used as a control, to exclude the possibility that the result is because of dilution of the GAL4 protein with an increased number of UAS transgenes. 

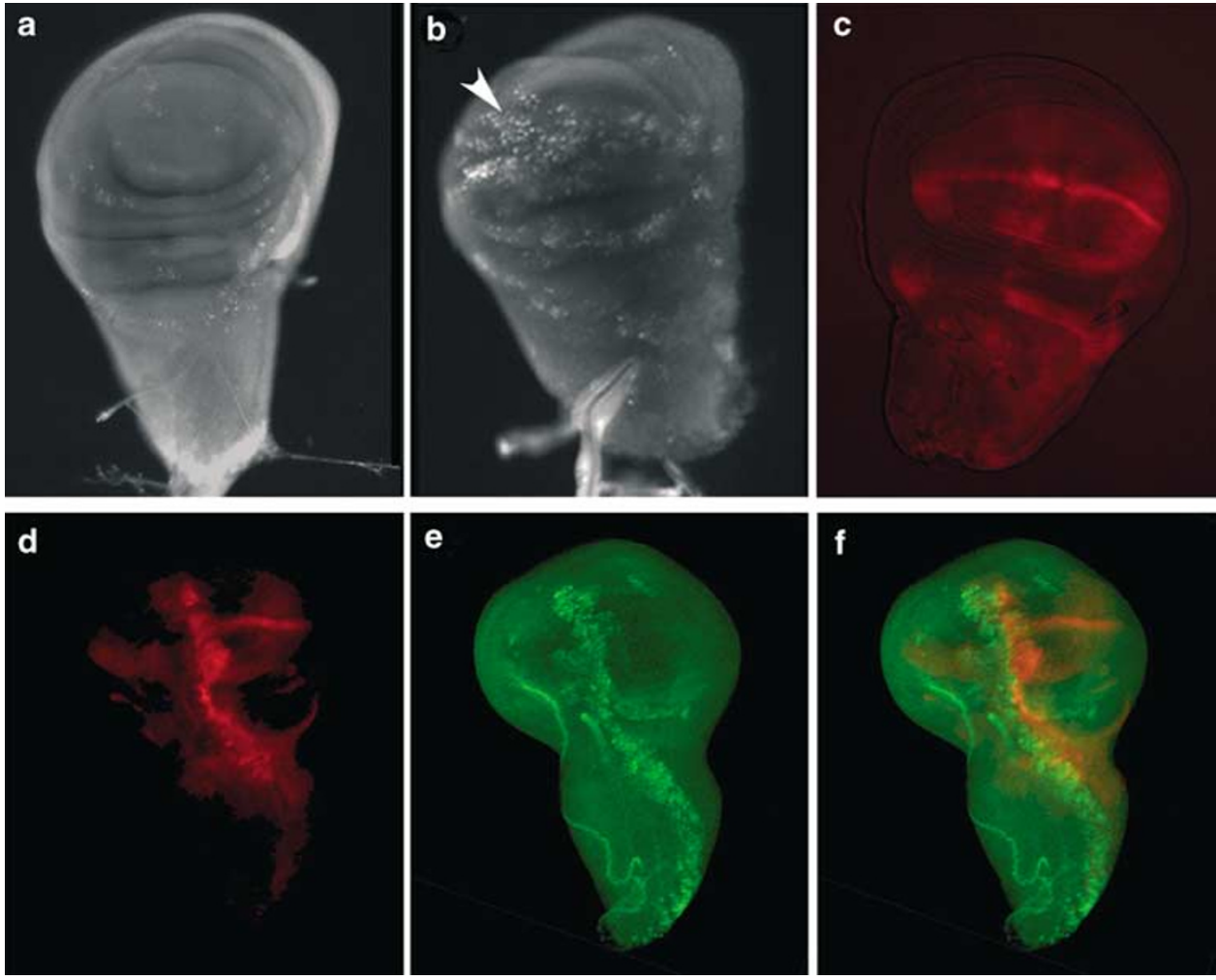

Figure 3 (a) Staining with acridine orange of a wild-type wing disc; no apoptosis is observed. (b) Staining with acridine orange of the wing disc of a F1 from the cross between UAS-twistD and MS1096-GAL4 flies. Apoptosis in the wing pouch where the driver is expressed is observed (white arrow head). (c) reaper-LacZ expression in a third instar wing disc of a wild-type strain using anti- $\beta$-gal antibody. Endogenous expression of reaper occurs in the wild-type strain mainly at the level of the future wing margin. (d) reaper-LacZ expression in the wing disc of a F1 from the cross between UAS-twistD and dpp-GAL4, reaper-LacZ flies, using anti- $\beta$-gal antibody. Increased reaper expression according to the driver is observed. (e) The same disc stained with anti-TWIST antibody. (f) Merge of (d) and (e). Only a slight overlapping is observed and the domain of expression of reaper is larger than that of twistD, suggesting a non-cell autonomous effect

Another way to evaluate a possible role of twistCE in proliferation was to express the UAS-twistCE transgene ectopically in a genetic context that affects cell proliferation. The $v g^{\text {null }}$ mutant phenotype is because of absence of proliferation at the wing margin. No apoptosis can be detected in wing imaginal discs of $v g^{\text {null }}$ mutants and overexpression of dacapo using $v g$ regulatory sequences in a $v g^{\text {null }}$ heterozygous background enhances the effects of dacapo, suggesting synergistic effects between the function of the two genes in cell proliferation. ${ }^{25}$

We ectopically expressed twistCE under the control of $v g$-GAL4, and compared the effects when the UAS-twistCE strain was crossed with the $v g$-GAL4, $v g^{\text {null }}$ strain. The $v g^{\text {null }} / \mathrm{vg}^{+}$heterozygotes display a wild-type phenotype. The ectopic expression of twistCE using the $v g$-GAL4 driver, in a $v g^{\text {null }}$ heterozygous background dramatically enhanced the phenotype observed in a $v^{+}$background (Figure $4 b, c$ ). This result strongly supports the hypothesis that the wing phenotype observed when twistCE is expressed in the wing disc is because of a significant decrease in cell proliferation.

The effect of twistCE on cell proliferation was further analyzed by in situ staining using anti-BrdU antibody that stains $S$ phase cells (not shown) and by anti-phospho-histone H3 antibody that stains M phase cells (Figure 4d,e). When the
twistCE transgene was overexpressed in either the wing or the eye imaginal discs (MS1096-GAL4 and eye-GAL4 drivers, respectively), no significant difference in BrdU staining was observed compared to the control indicating that the level of $S$ phase cells was not sufficiantly altered to be detected by this method.

To evaluate an effect on mitotic cells, we then compared the number of M phase cells in the area defined by MS1096 expression, by double staining with anti-phospho-histone $\mathrm{H} 3$ and anti- $\beta$-galactosidase antibodies, with or without twistCE ectopic expresssion. We used this driver, as it allows expression in the whole wing pouch, to increase statistical significance. The wing pouch of 10 discs from the progeny of each cross were counted, and we found an average of $47 \mathrm{M}$ phase cells per disc for the control and only $18 \mathrm{M}$ phase cells per disc when twistCE was ectopically expressed $(65 \%$ reduction of $\mathrm{M}$ phase cells), confirming the role of the twistCE transgene on the rate of mitosis. This effect was restricted to the area where the driver is expressed. For instance, in the notum, there was no difference between the control cross and the flies expressing twistCE. Indeed, an average of $147 \mathrm{M}$ phase cells per notum were found for the Canton wild-type strain, and $141 \mathrm{M}$ phase cells per notum for the twistCE transgene expressing flies on 10 wing discs tested for each strain. 

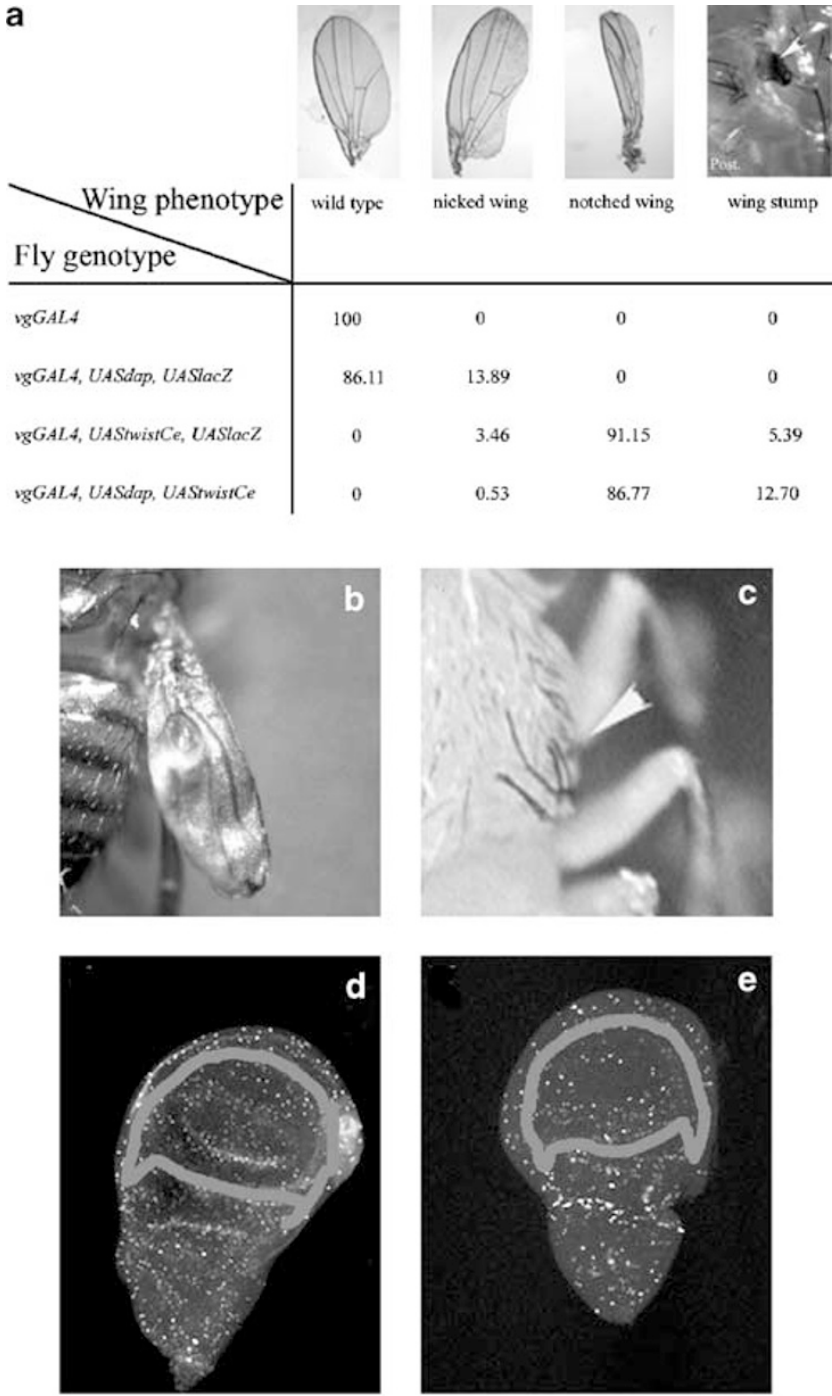

Figure 4 (a) Different wing phenotypes observed when dacapo, twistCE and both dacapo and twistCE are ectopically expressed in the wing disc using the $\mathrm{vg}$ GAL4 driver. The UAS-LacZ transgene was used to control for the possibility that the differences observed are because of dilution effects. In all, 500 wings were scored for each cross. (b)Wing of a F1 from the cross between UAS-twistCE and $v g$-GAL4 flies. A scalloped wing phenotype is observed. (c) Wing of a F1 from the cross between UAS-twistCE and $v g-G A L 4, v g^{\text {null }}$ flies. In this heterozygous $v g^{\text {nul }}$ background, a complete absence of the wing is observed (arrow for the normal place of the wing). (d) Anti-phospho-histone $\mathrm{H} 3$ staining of a third instar wing disc from the MS1096-GAL4 strain. The antibody stains cells in M phase (in red the limits of driver expression). (e) Anti-phospho-histone $\mathrm{H} 3$ staining of a third instar wing disc from the cross between UAS-twistCE and MS1096-GAL4 flies. A decrease in the number of $\mathrm{M}$ phase cells is observed according to the driver. We estimated the decrease in $\mathrm{M}$ phase in the wing pouch to be an average of $65 \%$

The fact that the transgene displays no effect using antiBrdU staining suggests a specific effect on the G2/M transition. A similar effect, but less spectacular, was observed using the 1151-GAL4 driver that allows expression in the myoblasts located on the notum of the wing imaginal disc (not shown).

Finally, the two other twist transgenes (UAS-twistD and UAS-twisth) did not decrease the number of $\mathrm{M}$ phase cells (data not shown). This indicates specificity in the effects of the different TWIST proteins: induction of apoptosis for twistD and a decrease in the mitotic rate for twistCE.

\section{Ectopic expression of human twist inhibits p53-dependent apoptosis}

Ectopic expression of human twist did not induce a wing phenotype. In mammals, it has been reported that twist can be considered a proto-oncogene that acts by inhibiting p53dependent apoptosis. ${ }^{19}$

Conservation in Drosophila of the function of human twist would indicate conservation between the human and Drosophila p53 pathways and that human twist cooperates with the equivalent Drosophila components of the pathway, and activates Drosophila targets that are homologs of human p53 targets.

In Drosophila, it has been shown that p53 activates the reaperpromoter but is unable to stop proliferation, and has no effect on dacapo, the p21 homolog. ${ }^{26,27}$

In order to test the effects of human twist on apoptosis, we decided to compare the effects of the twist $H$ transgene to the effects of a transgene encoding a dominant-negative form of Drosophila p53. This transgene (called here UAS-p53*) has been shown to inhibit apoptosis induced by ionizing radiation. ${ }^{26,27}$

To facilitate the evaluation of the decrease in cell death induced by the dominant-negative UAS-p53* or by the UAStwist $H$ transgene, we decided to express the transgenes in the wing disc and discriminate the effects on the change in wing phenotype compared to a control.

However, in the wing disc, massive cell death does not automatically lead to a wing phenotype, and only death of cells that will give the future wing margin affects wing morphology. Indeed, no adult wing phenotype were observed after exposure of larvae to ionizing radiation or UV, although acridine orange staining was clearly positive (data not shown).

Aminopterin, an analog of methotrexate, induces massive cell death and a well characterized wing phenotype when cell death occurs in the part of the wing disc that will give the future wing margin. ${ }^{28}$ This drug is an inhibitor of dihydrofolate reductase activity, and therefore inhibits DNA replication and the $\mathrm{G} 1 / \mathrm{S}$ transition.

We first tested whether cell death induced by aminopterin is p53 dependent by using the dominant-negative $p 53^{*}$ to try to inhibit the effect of the drug. When the UAS-p53*transgene is ectopically expressed using the $v g-G A L 4$ driver, no wing phenotype is observed. On a concentration of aminopterin $(2 \mathrm{mg} / \mathrm{kg})$ that induces nicks in the wings with the $\mathrm{vg}$-GAL4 strain that serves as control, the transgene counteracts to some extent the effect of the drug (51.4\% notched wings, $n=600$ for the control versus $40.2 \%$ notched wings, $n=616$ for p53*-expressing flies).

To enhance the effects of the aminopterin, we used a heterozygous $v g^{\text {null }}$ mutant context that is completely recessive on normal medium, but induces a high proportion of notched wings on a low concentration of aminopterin $(0.5 \mathrm{mg} /$ $\mathrm{kg}$ ) (Figure 5a). As expected, the effect of the UAS-p53* transgene is more spectacular in a $v g$-GAL4; $v g^{\text {null }} / \mathrm{vg}^{+}$ genotype (Figure 5a) and significant differences were 
a

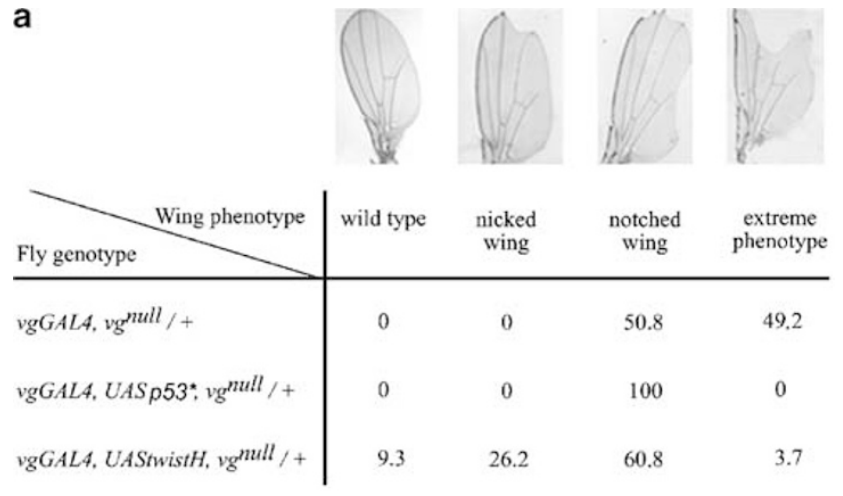

b

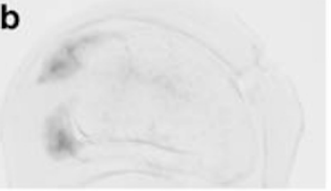

d

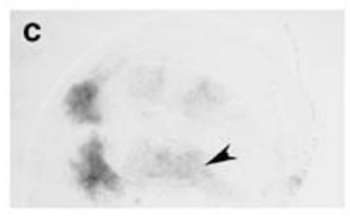

e

Figure 5 (a) The human twist transgene restores the wing phenotype from the effects of aminopterin. Proportion of the different wing phenotypes observed when $+/ v g^{\text {null }}$ flies, carrying different transgenes combinations, are reared on aminopterin $(1 \mathrm{mg} / \mathrm{kg})$. In the control $\left(v g-G A L 4,+/ v g^{n u l l}\right)$, half of the flies have 'notched' wings and half of a more 'extreme' phenotype when reared on aminopterin. Overexpression of UAS-p53* using $v g G A L 4$ sequences shifts the phenotype of $\mathrm{vg}$ null/+ heterozygotes from the effect of aminopterin towards only the 'notched' wings phenotype. The effect of the twistH transgene on $v g^{\text {null }}$ heterozygotes is more pronounced than with the UAS-p53* transgene, since 'nicked' wing and 'wild-type' wing phenotypes appear. (b) Expression of the $150 \mathrm{bp}$ reaper-LacZ construction in the wing disc of third instar larvae on normal medium. The same condition for $\beta$-gal staining were applied for $\mathbf{b}-\mathbf{e}$. (c) Expression of the $150 \mathrm{bp}$ reaper-LacZ in the wing disc of third instar larvae reared on aminopterin (1 mg/kg). An increase in staining is observed. (d) Effect of overexpression of UAS-p53* using the MS1096-GAL4 driver on the $150 \mathrm{bp}$ reaper$\mathrm{LaCZ}$ expression in the wing disc of third instar larvae reared on aminopterin $(1 \mathrm{mg} / \mathrm{kg})$. A decrease in the intensity of the staining is observed. (e) Effect of overexpression of UAS-twistH using the MS1096-GAL4 driver on the $150 \mathrm{bp}$ reaper-LacZ expression in the wing disc of third instar larvae reared on aminopterin $(1 \mathrm{mg} / \mathrm{kg})$. As for UAS-p53* expression, compared to $\mathrm{C}$, a decrease in the staining is observed

observed when compared to the control ( $v g-G A L 4 ; v^{\text {null }} / \mathrm{vg}^{+}$ strain). These results indicate that apoptosis induced by aminopterin is p53 dependent, and that this drug can be used to evaluate p53 activity.

The UAS-twistH transgene was also ectopically expressed using the $v g$-GAL4 driver first in a $v g^{+}$context. The $\mathrm{F} 1$ was reared on aminopterin $(2 \mathrm{mg} / \mathrm{kg})$, and we observed $42 \%$ notched wings $\left(n=376\right.$ ) compared to $v g-G A L 4 ; v^{+} / v g^{+}$flies that served as control $(51.4 \%, n=600)$. These results show that, as with the UAS-p53*transgene, UAS-TwistH reduced to some extent the effect of aminopterin.

Next, the UAS-twistH transgene was expressed using the $v g$-GAL4 driver in a $v g^{\text {null }}$ heterozygous context. The F1 was reared on aminopterin $(0.5 \mathrm{mg} / \mathrm{kg})$ and the wing phenotype compared to that of $v g$-GAL4; $\mathrm{vg}^{\text {null }} / \mathrm{vg}^{+}$ flies that served as a control for the experiment with the UAS-p53* transgene. As for UAS-p53*, a significant difference was observed compared to the control (Figure $5 a)$, suggesting that the human twist transgene shares properties with UAS-p53*. In fact, the UAS-twistH transgene is significantly more potent, shifting the wings more towards a wild-type phenotype than UAS-p53* (Figure 5a). The effect observed is not because of the genetic $v g$ context as it was observed in both a $v g^{+}$and a $v g^{\text {null }}$ heterozygous backgrounds.

The next step was to determine whether the effect of UAS-twistH is p53 dependent. p53-dependent apoptosis is because of the direct binding of p53 to the reaper promoter. It has been shown that a sequence of $150 \mathrm{bp}$ in the reaper promoter responds specifically to activation by $p 53 .^{26}$ We observed that a transgene that will be called here $150 \mathrm{bp}$ reaper-LacZ (a gift of $\mathrm{J}$ Abrams) is activated by p53 in the wing imaginal discs when flies are reared on aminopterin (Figure $5 \mathrm{~b}, \mathrm{c}$ ). In the wing disc of the $\mathrm{F} 1$ of the cross between UAS-p53* and MS1096-GAL4, 150 bp reaper$L a c Z$ flies raised on aminopterin, as expected, a significant diminution of $X$-Gal staining was observed in the wing pouch (Figure $5 d$ ). This confirms that the dominant-negative form of p53* disrupts activation of reaper transcription by active endogenous p53 via the $150 \mathrm{bp}$ sequence from the reaper promoter.

The same cross was done using the UAS-twist H transgene instead of UAS-p53* with the same result, indicating that the TWISTH product counteracts the active wild-type Drosophila p53 (Figure 5e). However, we cannot exclude the possibility that repression of the $150 \mathrm{bp}$ reaper-LacZtransgene by twistH is not due to the effect on p53 but rather to the effect on other factors that bind this reaper regulatory element. Another LacZ strain, called the $p 53$ response element has been described, which consists of oligomers of the p53 binding sites in the reaper promoter region. ${ }^{26}$ However, it cannot be used in the wing disc as it is not activated in this tissue by p53 (data not shown). The possibility that the Drosophila and nematode transgenes induce a similar effect was tested without positive results, indicating that the results were specific to the human transgene.

Taken together, these results strongly suggest that human twist, expressed in Drosophila, retains some of the same properties it has in human cells, in particular inhibition of p53dependent apoptosis. Moreover, in Drosophila, it acts on the reaper promoter.

\section{Ectopic expression of human twist induces cell proliferation}

It has been shown that vertebrate twist is able to induce proliferation of sketeletal muscle satellite cells. ${ }^{29}$ However, a clear role for vertebrate twist in proliferation has not been well established. Using our heterologous system, we tested if the human twist transgene is able to induce proliferation in Drosophila.

For this, we compared the wing phenotypes induced in the F1 of the cross between UAS-dacapo, UAS-LacZ, and $\mathrm{vg}$ - 
GAL4, $v g^{\text {null }}$ flies to those in the $\mathrm{F} 1$ of the cross between UASdacapo, UAS-twistH, and $v g-G A L 4, v g^{\text {null }}$ flies. In this $v g^{\text {null }}$ heterozygous background, nicks in the wing were observed in the former cross (Figure 6a), and this was not due to apoptosis but to inhibition of cell proliferation. ${ }^{24}$ In the latter cross, a significant rescue of the wing phenotype was observed, indicating that human twist can partially supress the dacapo overexpression phenotype, and thus increases cell proliferation and/or cell growth.

To confirm this result, we stained the wing disc with antiphospho-histone $\mathrm{H} 3$ antibody that marks cells in mitosis. An increase in staining was observed when human twist was expressed along with dacapo (UAS-twistH, UAS-dacapo) using the vg-GAL4 driver compared to the control (UAS-
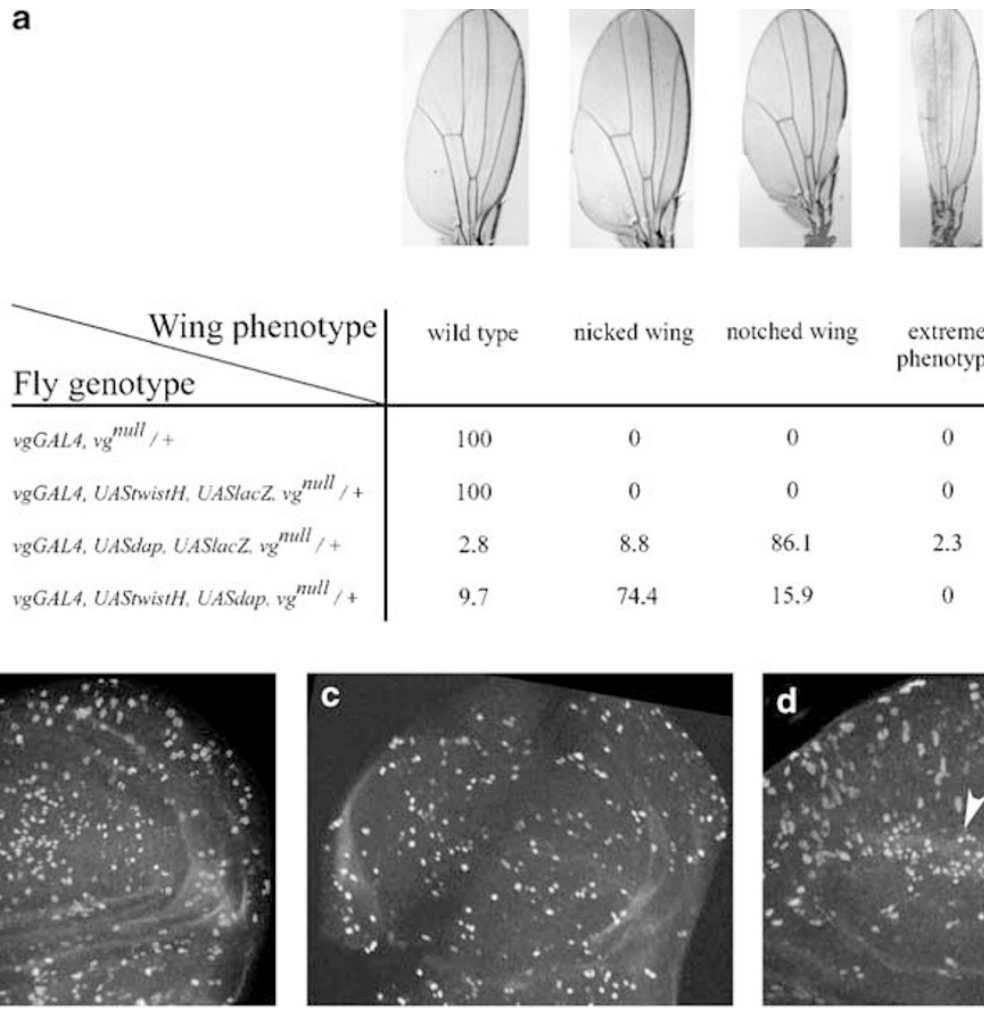

wild type

nicked wing notched wing

extreme

phenotype
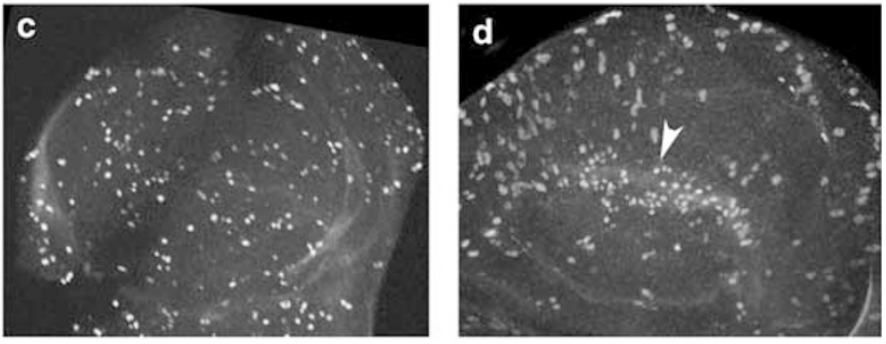

e

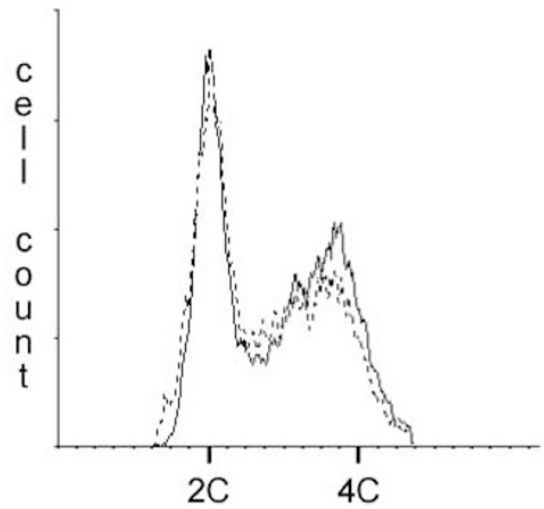

f

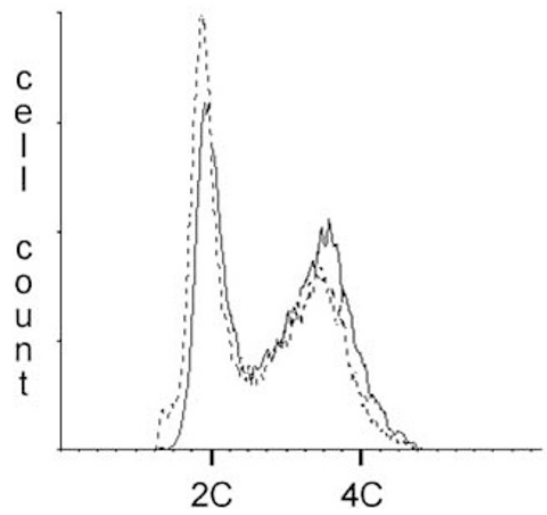

Figure 6 (a) The twist H transgene is an antagonist of the overexpression of dacapo. In the control $v g-G A L 4, v g^{\text {null }} /+$ flies or when $t$ twist $H$ is overexpressed using the $v g$ GAL4, only a wild-type wing phenotype is observed. Ectopic expression of dacapo or twistH and both dacapo and twistH in the wing disc using the $v g-G A L 4$ driver in a $\mathrm{vg}^{\text {null }}$ heterozygous background was assayed. Coexpression of dacapo and twist $H$ restores the wing phenotype induced by dacapo towards a 'nicked' wing phenotype instead of a 'notched' phenotype. (b) Anti-phospho-histone $\mathrm{H} 3$ staining of cells in $\mathrm{M}$ phase in a third instar wing imaginal disc of the $\mathrm{vg}^{\text {null }}$ heterozygous control strain. (c) Anti- phospho-histone $\mathrm{H} 3$ staining of a third instar wing imaginal disc of a F1 from the cross between UAS-dacapo and $v g$-GAL4 flies, in a $v g^{\text {null }} /+$ background. A decrease in staining in the wing pouch is observed. (d) Anti- phospho-histone $\mathrm{H} 3$ staining of a third instar wing imaginal disc of a F1 from the cross between UAS-dacapo, UAStwistH and $v g-G A L 4$ flies, in a $v g^{\text {null }}+$ background. An increase in cell undergoing mitosis is observed where the driver is expressed, compared to C (arrow head). (e-f) Flow cytometry. Uninterrupted and dotted lines represent $\mathrm{GFP}^{+}$(transgene-expressing posterior compartment) and GFP ${ }^{-}$(control anterior compartment) cells, respectively. Each trace is normalized to fit the graph as the number of $\mathrm{GFP}^{+}$and $\mathrm{GFP}^{-}$cells analyzed for each sample were not exactly equal. (e) Control: cells from third instar larvae wing imaginal discs from the cross between UAS-lacZ and en-GAL4, UAS-GFP flies. (f) Overexpression of the human twist transgene: cells from third instar larvae wing imaginal discs from the cross between UAS-twistH and en-GAL4, UAS-GFP flies 
dacapo, UAS-LacZ) showing that human twist drives cells in mitosis (Figure 6b-d).

To evaluate the rate of cell proliferation, we used the fluorescence-activated cell sorter (FACS). We performed FACS analysis using an en-GAL4; UAS-GFP line. This strain expresses the green fluorescent protein (GFP) under control of engrailed regulatory sequences in the posterior compartment of the wing disc, and so allows us to distinguish between wild-type cells and transgene-expressing cells. ${ }^{30}$ We compared the $\mathrm{F} 1$ from the cross between UAS-LacZ and enGAL4; UAS-GFP flies, which serves as an external control, to the $\mathrm{F} 1$ from the cross between UAS-twistH and en-GAL4; UAS-GFP flies. We used wing disc from larvae precisely staged at $120 \mathrm{~h} \mathrm{AED} \mathrm{(after} \mathrm{egg} \mathrm{deposit)} \mathrm{to} \mathrm{measure} \mathrm{the}$ proportion of cells in $\mathrm{G} 1, \mathrm{~S}$ and $\mathrm{G} 2 / \mathrm{M}$ phases in the two crosses. We compared cells from the posterior compartment (GFP ${ }^{+}$, transgene-overexpressing compartment) to cells from the anterior compartment $\left(\mathrm{GFP}^{-}\right.$, internal control compartment). Two sets of experiments were performed independantly, and data were analyzed using the Multicycle software. The control cross confirms that there is no significant difference between the two compartments because of the expression of a transgene in only one of the two compartments (Figure 6e). Indeed, the mean percentage change of $\mathrm{GFP}^{+}$cells compared with $\mathrm{GFP}^{-}$cells in the proportion of cells in $\mathrm{G} 1$ phase is a decrease of $3.05 \%$ for control experiments (Figure 6e, Table 1), which is not statistically significant. Conversely, the mean percentage change of cells in G1 is of $9.65 \%$ for UAS-twistH overexpression experiments (Figure 6f, Table 1), which is significant $(P<0.01)$. Moreover, the mean decrease in the proportion of $\mathrm{G} 1$ cells in the control cross is significantly different from that in the twistHoverexpressing cross $(P<0.1)$, and the mean increase of G2/M cells proportion is also significantly different $(P<0.1)$ (Table 1). However, no significant difference can be observed considering the proportion of $S$ phase cells. Those results indicate that the twist $H$ transgene causes a shift from the $\mathrm{G} 1$ phase to the G2/M phase of cell cycle, and so is an activator of cell proliferation.

Furthermore, the analysis of the Forward Scafold data, which is a measure of cell size, reveals no difference in average cell size between the two compartments in overexpression experiments (data not shown). This is surprising as overproliferation is generally accompanied by a reduction in cell size. But in fact, this could be explained if TWISTH not only activates cell proliferation but also promotes cell growth.

Table 1 Table shows the mean percentages in two experiments, for GFP control cells and $\mathrm{GFP}^{+}$transgene-expressing cells, and the mean changes between $\mathrm{GFP}^{-}$and $\mathrm{GFP}^{+}$cells

\begin{tabular}{|c|c|c|c|c|c|c|}
\hline & \multicolumn{4}{|c|}{ Mean percentage } & \multicolumn{2}{|c|}{ Mean change } \\
\hline & \multicolumn{2}{|l|}{ GFP $^{-}$} & \multicolumn{2}{|c|}{ GFP $^{+}$} & \multirow[b]{2}{*}{$\Delta \mathbf{G 1}$} & \multirow[b]{2}{*}{$\Delta \mathbf{G} 2$} \\
\hline & G1 & G2/M & G1 & G2/M & & \\
\hline Control & 33.15 & 34.35 & 30.1 & 36.7 & -3.05 & +2.35 \\
\hline $\begin{array}{l}\text { Human twist } \\
\text { overexpression }\end{array}$ & 35.55 & 32.55 & 25.9 & 48.3 & -9.65 & +15.75 \\
\hline
\end{tabular}

These data indicate that human twist retains, in Drosophila, the proto-oncogenic properties that have been described in vertebrate cultured cells, decreasing p53-dependent cell death and inducing proliferation. Even if the p53 pathways are significantly different between human and Drosophila, the human TWIST protein retains its potentials when expressed in the fly thus further supporting its role as an inducer of cell proliferation, per se, even in an epithelial tissue like the wing disc.

\section{Discussion}

Numerous transgenic Drosophila strains have been engineered to express human gene to better understand their function (for a review, see Chan and Bonini ${ }^{21}$ and Link ${ }^{31}$ ). This has been the case, in particular, for genes involved in neurodegenerative diseases like Alzheimer's, ${ }^{32,33}$ Parkinson, ${ }^{34}$ polyglutamine disease, ${ }^{35}$ and amyotrophic lateral sclerosis. $^{36}$ Thus, we used this method to study the potentialities of human TWIST and unravel new putative functions of the TWIST proteins of other species. In addition, this approach provides a useful tool to isolate modifiers of the different phenotypes induced by the ectopic genes.

Ectopic expression of Drosophila twist induced apoptosis and activated reaper expression. In contrast, ectopic expression of twistCE in the same cells did not induce apoptosis, but an arrest of proliferation that mimics the effect of dacapo, the homolog of $\mathrm{p} 21$.

The induction of apoptosis by twist $D$ in epithelial cells cannot be considered only as the result of an overload of ectopic protein, as neither twistCE nor twistH induce apoptosis. We showed that twistD has the potentiality to activate the proapoptotic pathway in Drosophila, clearly at least repear expression. The effect seems to be noncell autonomous, suggesting that it is not a direct effect on the reaper promoter as has been shown for Deformed, another developmental gene. ${ }^{37}$ The question now is if this potentiality of $t w i s t D$ is used during development or if it is only because of an ectopic effect. The fact that twist is expressed in proliferating myoblasts is an argument for a possible role in apoptosis in deregulated conditions. This could be linked to the observation of degeneration of the indirect flight muscles in twist hypomorph or when twist is overexpressed in the myoblasts. It has been suggested that degeneration is caused by apoptosis. ${ }^{23}$ This point will require further investigation.

twistH is able to act on the apoptotic pathway by repressing p53-dependent reaper activation. We provide several lines of evidence indicating that $t w i s t H$ is a suppressor of p53dependent apoptosis: (i) its ectopic expression induces the same effect as that of a dominant negative form of $p 53^{*}$ and (ii) it represses the $150 \mathrm{bp}$ reaper regulatory element that is normally activated by wild-type p53. This suggests that ectopic expression of twistH perturbs Drosophila p53 activation of some of its natural targets, at least reaper. This indicates that the potentialities of the TWIST protein are not restricted to one cell type and that the capacity for human TWIST to inhibit p53-dependent apoptosis can occur in vivo as we were able to reproduce it in Drosophila. The capacity to repress the apoptotic pathway in human cells has also been 
described for DermoD, which presents extensive homology with twistH principally at the level of the bHLH domain. This suggests that this domain is implicated in the phenomenon. ${ }^{19}$

However, here the effect observed is on Drosophila p53. It has been reported that if Drosophila p53 shares with mammalian p53 the capacity to respond to DNA damage via induction of apoptosis, it seems unable to induce a G1 arrest by activating dacapo, the Drosophila p21 homolog. ${ }^{26,27}$

Another important difference between Drosophila and mammalian p53 is that the residues critical for MDM2 binding are not conserved in Drosophila p53. ${ }^{27}$ MDM2 is a potent negative regulator of p53 that acts by binding to, and thus inhibiting, the p53 transactivation domain and by fostering p53 degradation. ${ }^{38,39}$ Stabilization of p53 occurs when the ARF tumor suppressor interferes with MDM2 function. ${ }^{40}$ ARF binds directly to MDM2 thus enabling transcriptionally active p53 to accumulate in the nucleoplasm. ${ }^{41,42}$

It has been suggested that the effects of the ectopic expression of twistH on p53-dependent apoptosis in cultured cells are mediated by the effects of twist on ARF. ${ }^{19}$ Indeed, transient expression of twistH induces a dramatic downregulation of ARF, thus providing a potential mechanism by which twist may affect p53 function. However, since reaper is the only identified target of Drosophila p53 at the moment, it is difficult to explore further how $t w i s t H$ could directly counteract Drosophila p53.

Our results indicate conservation of $t w i s t H$ potentialities in Drosophila. This suggests that either: (i) in fact, the ARF/ MDM2/p53 pathway exists in Drosophila but ARF and MDM2 have not been identified yet or (ii) twistH acts on Drosophila p53 activity by affecting an alternative pathway. Indeed, twistH has been shown to interact in vitro with P300/CBP, regulating its histone acetyltransferase activity, and thus could interfere with p53 activity altering its ability to bind target sequences in vitro. ${ }^{43}$

Moreover, recently, it was shown that the antiapoptotic action of insulin-like growth factor (IGF-1) signaling is mediated by induction of twist suggesting a key role for Mammalian twist as an antiapoptotic factor. ${ }^{20}$ Thus, a link between the antiapoptotic action of the IGF-1 receptor and p53-dependent apoptosis via twist can be suggested.

In this study, we also considered Twist potentialities on cell proliferation. We showed that both twistCE and twistH could act on proliferation. Ectopic expression of twistCE induces an arrest of proliferation. This was clearly observed using an antiphospho-histone $\mathrm{H} 3$ antibody that stains mitotic cells, while no effect was observed with anti-BrdU antibody that marks cells in $\mathrm{S}$ phase suggesting the possibility that the G2/M transition is principally affected. In addition, ectopic expression of twistCE aggravated the reduced wing phenotype induced by dacapo and by a decrease in VG protein dose, confirming that TwistCE can antagonize the normal mitotic process. The fact that TwistCE is expressed only in differentiated mesodermic tissue can perhaps be linked to our results. ${ }^{11}$

In contrast, twistH is able to increase epithelial cell proliferation in Drosophila. FACs analysis showed that ectopic expression of human twist shifts the repartition of the cells toward a decrease in the percentage of cells in $\mathrm{G} 1$ to an increase in the percentage of cells $\mathrm{G} 2 / \mathrm{M}$, suggesting an effect of TWISTH principally on mitosis. Such a proliferative function has not been clearly reported so far in mammalian cultured cells or in in vivo system. However, it has been shown that hepatocyte growth factor (HGF) inhibits skeletal muscle cell differentiation and stimulates cell proliferation of satellite cells probably by inducing twist expression. ${ }^{29}$ Interestingly, IGF-1 can also promote proliferation of satellite cells, ${ }^{44}$ thus linking the role of vertebrate twist in p53-dependent apoptosis and proliferation.

On the whole, the transgenic strains expressing ectopic twist enable us to establish different potentialities for twistD and twistCE that can now be investigated to determine whether twist $D$ induction of apoptosis and twistCE inhibition of mitotic activity occur naturally during the developmental processes where twist is required. To identify which domains of the TWIST protein are responsible for the phenotypes observed, chimeric transgenic lines where bHLH domain and/or the WR motif of one species would be replaced by that of another should prove informative. Other domains of the TWIST protein can also be analyzed in this way.

Most important, we show that the role of twistH as a potential oncogene can be studied in vivo using Drosophila transgenic lines suggesting that the targets of p53-dependent apoptosis and induction of proliferation are conserved in Drosophila. This gives us a powerful tool to screen for targets and for partners of the p53 and proliferation pathways, as well as modifiers.

\section{Materials and Methods}

\section{Generation of trangenics flies}

UAS-twistH and UAS-twistCE strains were constructed in our laboratory by transgenesis. ${ }^{45}$ The twist cDNAs, gifts from Fabienne Perrin-Shmid ${ }^{46}$ and Andrew Fire, ${ }^{10}$ respectivily, were cloned into pCAsPeR vector. ${ }^{47}$ Five independent lines were obtained with the human transgene, and three with the nematode transgene. For both transgenes, all lines give a similar result.

\section{Fly stocks}

The Drosophila UAS-twist line (UAS-twistD) was provided by Mary Baylies. ${ }^{7}$ UAS-p53* was a gift from Dr. Kopczynski. ${ }^{27}$

The different GAL4 drivers used were obtained from the Bloomington stock center. GMR-GAL4 expression is restricted to the presumptive photoreceptor cells posterior to the morphogenetic furrow of the eye imaginal disc, whereas eye-GAL4 is expressed in the entire eye-antenna imaginal disc, and thus usually leads to more drastic effects. The dppGAL4 strain (Figure 2a) was used to drive expression in both eye imaginal discs, specifically in the morphogenetic furrow, and wing imaginal discs, specifically at the anteroposterior compartment boundary. The vg-GAL4 driver, which is expressed at the dorso-ventral compartment boundary (Figure 2a), allows us to express the transgenes at the level of the future wing margin that is very sensitive to a decrease in cell number, and thus can induce an easily visible wing phenotype. The MS1096-GAL4 driver (Figure 2a), which drives expression in the entire wing pouch, and also displays a faint and variable expression in the notum, was used to conduct statistical experiments on proliferation rates in the wing pouch, as it gives a larger expression domain than other wing drivers. The 1151 - 
GAL4 driver allows expression in the myoblasts located on the notum of the wing disc.

The reporter construct strains were provided by Dr. Abrams. ${ }^{26}$ The reaper-LacZ strain carries the $L a c Z$ sequences under the control of the entire promoter of the reaper gene; the $150 \mathrm{bp}$ reaper-LacZ reports the activation of the $150 \mathrm{bp}$ of the reaper promoter that respond to the Drosophila p53 protein.

We also used the vestigial null mutant, named $v g^{\text {null }}$. The $v g^{\text {null }}$ mutant leads to absence of proliferation at the wing margin, and displays complete absence of wings in homozygous adults. ${ }^{48}$

\section{Acridine orange staining}

Detection of apoptotic cells was performed with acridine orange staining. Third instar larvae were dissected, and imaginal discs were collected in PBS $1 \times$. Imaginal discs were then incubated $2 \mathrm{~min}$ in a $0.6 \mu \mathrm{g} / \mathrm{ml}^{1}$ acridine orange/PBS $1 \times$ solution and rinsed very briefly in PBS $1 \times$ before observation.

\section{Histochemical detection}

LacZ staining was performed on dissected wing imaginal disc. Third instar larvae were dissected in PBS $1 \times$, fixed in $4 \%$ formaldehyde- $1 \%$ glutaraldehyde and wing discs were incubated in a $0.04 \% \mathrm{X}$-gal solution overnight. All genotypes were treated simultaneously to avoid variation because of different experiments.

Immunostaining was performed as previously described. ${ }^{25}$ Antibody against Drosophila TWIST protein was a gift from S Roth ${ }^{49}$ and was used at $1 / 5000$ dilution. Antibody against human TWIST protein is avalaible at Santa Cruz Biotechnology and used at 1/200 concentration. Anti-phosphohistone $\mathrm{H} 3$ stains specifically phosphorylated histone 3 , and so allows detection of cells in mitosis; anti-phospho-histone $\mathrm{H} 3$ antibody is available at Upstate Biotechnology used at 1/2000 concentration. Anti- $\beta$-gal antibody (from Amersham) is a mouse antibody and was used at 1/200 dilution.

\section{Fluorescence-activated cell sorter (FACS) experiments}

Staged larvae of $120 \mathrm{~h} \mathrm{AED}$, derived from 2 to $3 \mathrm{~h}$ egg collections, and raised at $25^{\circ} \mathrm{C}$ were dissected in PBS. In all, 20 wing discs were washed twice in PBS and incubated with gentle agitation for $150 \mathrm{~min}$ according to Neumann and Edgar. ${ }^{50}$ An Elite Beckman Coulter FACS was used, and data were analyzed using the Multicycle Software.

\section{Acknowledgements}

The authors are very grateful to M Baylies, A Fire, J Abrams, F PerrinSchmidtt for fly stocks and cDNA. They thank $J$ Abrams for helpful comments. They acknowledge the excellent technical assistance of $A$ Dutriaux, B Legois, and MC Gendron. The work was supported by the $A R C$ and $A T C$ vieillissement.

\section{References}

1. Yun K and Wold B (1996) Skeletal muscle determination and differentiation: story of a core regulatory network and its context. Curr. Opin. Cell Biol. 8: 877889
2. Murre C, McCaw PS, Vaessin H, Caudy M, Jan LY, Jan YN, Cabrera CV, Buskin JN, Hauschka SD, Lassar AB et al. (1989) Interactions between heterologous helix-loop-helix proteins generate complexes that bind specifically to a common DNA sequence. Cell. 58: 537-544

3. Leptin M (1991) Twist and snail as positive and negative regulators during Drosophila mesoderm development. Genes Dev. 5: 1568-1576

4. Castanon I and Baylies MK (2002) A Twist in fate: evolutionary comparison of Twist structure and function. Gene 287: 11-22

5. Simpson P (1983) Maternal-zygotic gene interactions during formation of the dorsoventral pattern in Drosophila embryos. Genetics 105: 31-40

6. Thisse B, Stoetzel C, Gorostiza-Thisse C and Perrin-Schmitt F (1988) Sequence of the twist gene and nuclear localization of its protein in endomesodermal cells of early Drosophila embryos. EMBO J. 7: 2175-2183

7. Baylies MK and Bate M (1996) Twist, a myogenic switch in Drosophila. Science 272: $1481-1484$

8. Bate M, Rushton E and Currie DA (1991) Cells with persistent twist expression are embryonic precursors of adult muscles in Drosophila. Development 110: 791-804

9. Currie DA and Bate M (1991) The development of adult abdominal muscles in Drosophila: myoblasts express twist and are associated with nerves. Development 113: 91-102

10. Harfe BD, Vaz Gomez A, Kenyon C, Liu J, Krause M and Fire A (1998) Analysis of a Caenorhabditis elegans Twist homolog identifies conserved and divergent aspects of mesodermal patterning. Genes Dev. 12: 2623-2635

11. Corsi AK, Kostas SA, Fire A and Krause M (2000) Caenorhabditis elegans twist plays an essential role in non-striated muscle development. Development 127: 2041-2051

12. Hebrok M, Wertz K and Füchtbauer EM (1994) M-twist is an inhibitor of muscle differentiation. Dev. Bio. 165: 537-544

13. Spicer DB, Rhee J, Cheung WL and Lassar AB (1996) Inhibition of myogenic bHLH and MEF2 transcription factors by the bHLH protein Twist. Science 272: 1476-1480

14. Chen ZF and Behringer RR (1995) Twist is required in head mesenchyme for cranial neural tube morphogenesis. Genes Dev. 9: 686-699

15. Howard TD, Paznekas WA, Green ED, Chiang LC, Ma N, Ortiz de Luna RI, Garcia Delgado C, Gonzales-Ramos M, Kline AD and Wang Jabs E (1997) Mutations in TWIST, a basic helix-loop-helix transcription factor, in SaethreChotzen syndrome. Nat Gen. 15: 36-41

16. El Ghouzzi V, Le Merrer M, Perrin-Shmitt F, Lajeunie E, Benit P, Renier D, Bourgeois P, Bolcato-Bellemin AL, Munnich A and Bonaventure J (1997) Mutations of the TWIST gene in the Saethre-Chotzen syndrome. Nat Genet. 15: $42-46$

17. Johnson D, Horsley SW, Moloney DM, Oldridge M, Twigg SR, Walsh S, Barrow M, Njolstad PR, Kunz J, Ashworth GJ, Wall SA, Kearney L and Wilkie AO (1998) A comprehensive screen for TWIST mutations in patients with craniosynostosis identifies a new microdeletion syndrome of chromosome band 7p21.1. Am. J. Hum. Genet. 63: 1282-1293

18. El Ghouzzi V, Legeai-Mallet L, Benoist-Lasselin C, Lajeunie E, Renier D, Munnich A and Bonaventure J (2001) Mutations in the basic domain and the loop-helix II junction of TWIST abolish DNA binding in Saethre-Chotzen syndrome. FEBS Lett. 492: 112-118

19. Maestro R, Dei Tos AP, Hamamori Y, Krasnokutsky S, Sartorelli V, Kedes L, Doglioni C, Beach DH and Hannon GJ (1999) Twist is a potential oncogene that inhibits apoptosis. Genes Dev. 13: 2207-2217

20. Dupont J, Fernandez AM, Glackin CA, Helman L and LeRoith D (2001) Insulinlike growth factor 1 (IGF-1)-induced twist expression is involved in the antiapoptotic effects of the IGF-1 receptor. J. Biol. Chem. 276: 26699-26707

21. Chan HY and Bonini NM (2000) Drosophila models of human neurodegenerative disease. Cell Death Differ. 7: 1075-1080

22. Castanon I, Von Stetina S, Kass J and Baylies MK (2001) Dimerization partners determine the activity of the Twist bHLH protein during Drosophila mesoderm development. Development 128: 3145-3159

23. Anant S, Roy S and VijayRaghavan K (1998) Twist and Notch negatively regulate adult muscle differentiation in Drosophila. Development 125: 13611369

24. Lane ME, Sauer K, Wallace K, Jan YN, Lehner CF and Vaessin H (1996) Dacapo, a cyclin-dependent kinase inhibitor, stops cell proliferation during Drosophila development. Cell 87: 1225-1235 
25. Van de Bor V, Delanoue R, Cossard R and Silber J (1999) Truncated products of the vestigial proliferation gene induce apoptosis. Cell Death Differ. 6: 557564

26. Brodsky MH, Nordstrom W, Tsang G, Kwan E, Rubin G and Abrams JM (2000) Drosophila p53 binds a damage response element at the reaper locus. Cell 101: 103-113

27. Ollmann M, Young LM, Di Como CJ, Karim F, Belvin M, Robertson S, Whittaker K, Demsky M, Fisher WW, Buchman A, Duyk G, Friedman L, Prives C and Kopczynski C (2000) Drosophila p53 is a structural and functional homolog of the tumor suppressor p53. Cell 101: 91-101

28. Silber J, Coste A, Bazin C and Le Menn A (1985) Dihydrofolate reductase activity and resistance to aminopterin in various species of Drosophila. Mol. Gen. Genet. 200: 92-95

29. Leshem Y, Spicer DB, Gal-Levi R and Halevy O (2000) Hepatocyte growth factor (HGF) inhibits skeletal muscle cell differentiation: a role for the bHLH protein twist and the cdk inhibitor p27. J. Cell Physiol. 184: 101-109

30. Britton JS, Lockwood WK, Li L, Cohen SM and Edgar BA (2002) Drosophila's insulin/PI3-kinase pathway coordinates cellular metabolism with nutritional conditions. Dev. Cell 2: 239-249

31. Link CD (2001) Transgenic invertebrate models of age-associated neurodegenerative diseases. Mech. Ageing Dev. 122: 1639-1649

32. Luo L, Tully T and White K (1992) Human amyloid precursor protein ameliorates behavioral deficit of flies deleted for Appl gene. Neuron 9: 595-605

33. Fossgreen A, Bruckner B, Czech C, Masters CL, Beyreuther K and Paro R (1998) Transgenic Drosophila expressing human amyloid precursor protein show gamma-secretase activity and a blistered-wing phenotype. Proc. Natl. Acad. Sci. 95: 13703-13708

34. Feany MB and Bender WW (2000) A Drosophila model of Parkinson's disease. Nature 404: 394-398

35. Warrick JM, Chan HY, Gray-Board GL, Chai Y, Paulson HL and Bonini NM (1999) Suppression of polyglutamine-mediated neurodegeneration in Drosophila by the molecular chaperone HSP70. Nat. Genet. 23: 425-428

36. Elia AJ, Parkes TL, Kirby K, St George-Hyslop P, Boulianne GL, Phillips JP and Hilliker AJ (1999) Expression of human FALS SOD in motorneurons of Drosophila. Free Radic. Biol. Med. 26: 1332-1338

37. Lohmann I, McGinnis N, Bodmer M and McGinnis W (2002) The Drosophila Hox gene deformed sculpts head morphology via direct regulation of the apoptosis activator reaper. Cell 110: 457-466
38. Momand J, Zambetti GP, Olson DC, George D and Levine AJ (1992) The mdm2 oncogene product forms a complex with the p53 protein and inhibits p53mediated transactivation. Cell 69: 1237-1245

39. Haupt Y, Maya R, Kazaz A and Oren M (1997) Mdm2 promotes the rapid degradation of $p 53$. Nature 387: 296-299

40. Sherr CJ and Weber JD (2000) The ARF/p53 pathway. Curr. Opin. Genet Dev. 10: $94-99$

41. Tao W and Levine AJ (1999) P19(ARF) stabilizes p53 by blocking nucleo-cytoplasmic shuttling of Mdm2. Proc. Natl. Acad. Sci. 96: 69376941

42. Weber JD, Jeffers JR, Rehg JE, Randle DH, Lozano G, Roussel MF, Sherr CJ and Zambetti GP (2000) p53-independent functions of the p19(ARF) tumor suppressor. Genes Dev. 14: 2358-2365

43. Hamamori Y, Sartorelli V, Ogryzko V, Puri PL, Wu HY, Wang JY, Nakatani Y and Kedes $L$ (1999) Regulation of histone acetyltransferases p300 and PCAF by the bHLH protein twist and adenoviral oncoprotein E1A. Cell 96: $405-413$

44. Allen RE and Boxhorn LK (1989) Regulation of skeletal muscle satellite cell proliferation and differentiation by transforming growth factor-beta, insulin-like growth factor I, and fibroblast growth factor. J. Cell Physiol. 138: 311-315

45. Spradling AC and Rubin GM (1982) Transposition of cloned P elements into Drosophila germ line chromosoms. Science 218: 341-347

46. Bourgeois $P$, Stoetzel $C$, Bolcato-Bellemin AL, Mattei MG and Perrin-Schmitt $F$ (1996) The human H-twist gene is located at 7p21 and encodes a B-HLH protein that is $96 \%$ similar to its murine M-twist counterpart. Mamm. Genome 7 : 915-917

47. Pirotta V (1988) Vectors for P-mediated transformation in Drosophila. Biotechnology 10: 437-456

48. Paumard-Rigal S, Zider A, Vaudin P and Silber S (1998) Specific interactions between vestigial and scalloped are required to promote wing tissue proliferation in Drosophila melanogaster. Dev. Genes Evol. 208: 440446

49. Roth S, Stein D and Nusslein-Volhard C (1989) A gradient of nuclear localization of the dorsal protein determines dorsoventral pattern in the Drosophila embryo. Cell 159: 1189-1202

50. Neufeld TP, de la Cruz AF, Johnston LA and Edgar BA (1998) Coordination of growth and cell division in the Drosophila wing. Cell 93: 1183-1193 